\title{
Implementation of High-Flow Nasal Cannula Therapy Outside the Intensive Care Setting
}

\author{
Julie A Jackson, Sarah K Spilman, Lisa K Kingery, Trevor W Oetting, Matthew J Taylor, \\ William M Pruett, Christopher R Omerza, Kaitlin A Branick, Iaswarya Ganapathiraju, \\ Mikayla Y Hamilton, Dakota A Nerland, Philip S Taber, Dustin A McCann, \\ Carlos A Pelaez, and Matthew W Trump
}

\begin{abstract}
BACKGROUND: High-flow nasal cannula (HFNC) is an option for respiratory support in patients with acute hypoxic respiratory failure. To improve patient outcomes, reduce ICU-associated costs, and ease ICU bed availability, a multi-phased, comprehensive strategy was implemented to make HFNC available outside the ICU under the supervision of pulmonology or trauma providers in cooperation with a dedicated respiratory therapy team. The purpose of this study was to describe the education and implementation process for initiating HFNC therapy outside the ICU and to convey key patient demographics and outcomes from the implementation period. METHODS: HFNC therapy was implemented at a tertiary hospital in the Midwest, with systematic roll-out to all inpatient floors over a 9-month period. Utilization of the therapy and patient outcomes were tracked to ensure safety and efficacy of the effort. RESULTS: During the implementation period, 346 unique subjects met study inclusion criteria. Median (interquartile range) hospital length of stay was $8 \mathrm{~d}$ (4-12), and median duration of HFNC therapy was $44 \mathrm{~h}$ (18-90). Two thirds of subjects $(n=238)$ received the entire course of HFNC therapy outside the ICU, and more than half of subjects $(n=184)$ avoided the ICU for their entire hospitalization. Moreover, $6 \%$ of subjects in the study group escalated from HFNC to noninvasive ventilation, and 5\% of subjects escalated from HFNC to mechanical ventilation. CONCLUSIONS: A comprehensive implementation process and a robust therapy protocol were integral to initiating and managing HFNC in all hospital locations. Study findings indicate that patients with acute hypoxic respiratory failure can safely receive HFNC therapy outside the ICU with appropriate patient selection and staff education. Key words: high-flow nasal cannula; intensive care unit; inpatient; emergency department; education; mechanical ventilation; noninvasive ventilation. [Respir Care 2021;66(3):357-365. (c) 2021 Daedalus Enterprises]
\end{abstract}

\section{Introduction}

For many decades, oxygen administration has been a common intervention to treat acute hypoxic respiratory failure by improving gas exchange and decreasing work of breathing. ${ }^{1-5}$ It is estimated that $40-60 \%$ of patients

Ms Jackson, Ms Kingery, and Mr Oetting are affiliated with Respiratory Therapy, UnityPoint Health, Des Moines, Iowa. Ms Spilman and Dr Pelaez are affiliated with Trauma Services, UnityPoint Health, Des Moines, Iowa. Dr Taylor is affiliated with the Division of Pulmonary, Critical Care, and Sleep Disorders Medicine Fellowship Program, University of Louisville, Louisville, Kentucky. Dr Pruett is affiliated with the Division of Pulmonary, Critical Care, and Sleep Disorders Medicine Fellowship Program, Creighton University, Omaha, Nebraska. admitted to hospitals will receive some form of oxygen administration during their stay. ${ }^{6,7}$ However, if patients develop worsening hypoxia, they often require admission to the ICU to receive more aggressive respiratory support. ${ }^{8}$ Hospitals struggle to have enough ICU beds available for critically ill patients, especially during high peak seasons such as influenza or during pandemics, and ICU costs are a

\footnotetext{
Dr Omerza is affiliated with the General Surgery Residency Program, UnityPoint Health, Des Moines, Iowa. Drs Branick and Ganapathiraju are affiliated with the Internal Medicine Residency Program, UnityPoint Health, Des Moines, Iowa. Ms Hamilton and Ms Nerland are affiliated with the Doctor of Osteopathic Medicine Program, Des Moines University, Des Moines, Iowa. Dr Taber is affiliated with Emergency Medicine, UnityPoint Health, Des Moines, Iowa. Dr McCann is
} 


\section{IMPLEMENTING HFNC OUTSIDE THE ICU}

significant component of total health care costs. ${ }^{9-13}$ It is essential to establish options for delivering safe and effective respiratory therapy to patients outside the ICU setting.

\section{See the Related Editorial on Page 531}

High-flow nasal cannula (HFNC) therapy has become an increasingly popular form of respiratory support for ICU patients with worsening hypoxic respiratory failure. ${ }^{14-21}$ There are many advantages of HFNC, including ease of use, patient comfort, and efficient delivery of humidified highflow oxygen. ${ }^{22-26}$ In many hospitals, HFNC is available only in the ICU setting, but this restriction can delay treatment if there are no available ICU beds and may result in a loweracuity patient being moved to the ICU only to receive treatment. Some hospitals have developed protocols for delivering HFNC on general in-patient pediatric floors ${ }^{27,28}$ or in the emergency department, ${ }^{29-31}$ but there is relatively little research establishing safety and efficacy in adult populations outside the ICU. Zemach et $\mathrm{al}^{32}$ conducted an observational study of subjects receiving HFNC in non-ICU areas and reported that HFNC was associated with improvements in breathing frequency and oxygen saturation.

Expanding HFNC availability outside the ICU requires careful and structured implementation of the therapy in new settings, often with staff who have not previously cared for patients on HFNC therapy. Introducing a new therapy is a challenge for any hospital, but successful implementation and buy-in from staff are essential for ensuring patient safety and quality of care throughout the hospital stay. ${ }^{33,34}$ The purpose of this study is to describe the education and implementation processes for delivering HFNC to non-ICU settings in our hospital. Specifically, we will describe the protocol and the implementation process, present key patient demographics and outcomes, and identify potential barriers that must be addressed to maximize patient safety.

affiliated with Pulmonary and Critical Care Medicine, US Department of Veteran's Affairs, Des Moines, Iowa. Dr Pelaez is affiliated with Trauma Surgery, The Iowa Clinic, West Des Moines, Iowa. Dr Trump is affiliated with Pulmonary and Critical Care Medicine, The Iowa Clinic, West Des Moines, Iowa. Dr Trump is affiliated with Pulmonary and Critical Care Medicine, UnityPoint Health, Des Moines, Iowa.

Ms Jackson presented a version of this paper at the 2019 AARC Congress, held November 9-12, 2019, in New Orleans, Louisiana. Ms Jackson and Dr Trump also presented at an industry-sponsored symposium on this topic at the 2019 AARC Congress.

Supplementary material related to this paper is available at http://www. rcjournal.com.

\section{QUICK LOOK}

\section{Current knowledge}

High-flow nasal cannula (HFNC) has been used extensively in the ICU setting for patients with hypoxic respiratory failure. There can be a high demand for ICU beds during peak periods, use of HFNC outside the ICU requires better understanding of risks and benefits.

\section{What this paper contributes to our knowledge}

Study findings indicate that HFNC can be delivered to patients outside the ICU if done so in the context of a broad implementation strategy and appropriate patient selection. Two thirds of study subjects at a tertiary hospital received the duration of HFNC therapy outside the ICU, and more than half of subjects avoided the ICU during hospitalization. HFNC was delivered safely and efficaciously to subjects with hypoxic respiratory failure.

\section{Methods}

\section{Study Design}

This study was conducted at Iowa Methodist Medical Center in Des Moines, Iowa, which is a tertiary hospital and Level 1 trauma center with 370 hospital beds, a 33-bed emergency department, and a 36-bed mixed medical, surgi$\mathrm{cal}$, and trauma ICU. HFNC has been used extensively in the adult ICU at the study hospital since 2012 and has been described in previous research. ${ }^{15,17,35}$ The research protocol was approved by the institutional review board at the study hospital.

During the 18-month study period (December 2017 to May 2019), HFNC therapy was primarily delivered with AIRVO2 (Fisher \& Paykel, Auckland, New Zealand), which can deliver flow up to $60 \mathrm{~L} / \mathrm{min}$ and $\mathrm{F}_{\mathrm{IO}_{2}}$ up to 1.0 . This device was selected because it does not require a compressed air supply and was therefore portable and

At the time of this study, Drs Pruett and Taylor were affiliated with the Internal Medicine Residency Program, and Dr McCann was affiliated with Pulmonary and Critical Care Medicine, UnityPoint Health, Des Moines, Iowa. Dr McCann was also affiliated with Pulmonary and Critical Care Medicine, The Iowa Clinic, West Des Moines, Iowa.

Fisher \& Paykel Healthcare provided grant support and donated disposables to UnityPoint Health to support nurse and physician education during the implementation phase of the study. Ms Jackson and Dr Trump have disclosed relationships with Fisher \& Paykel HealthCare. The other authors have disclosed no conflicts of interest.

Correspondence: Julie A Jackson RRT RRT-ACCS, UnityPoint Health, 1200 Pleasant St, Des Moines, IA 50309. E-mail: julie.jackson@unitypoint.org.

DOI: $10.4187 /$ respcare. 07960 


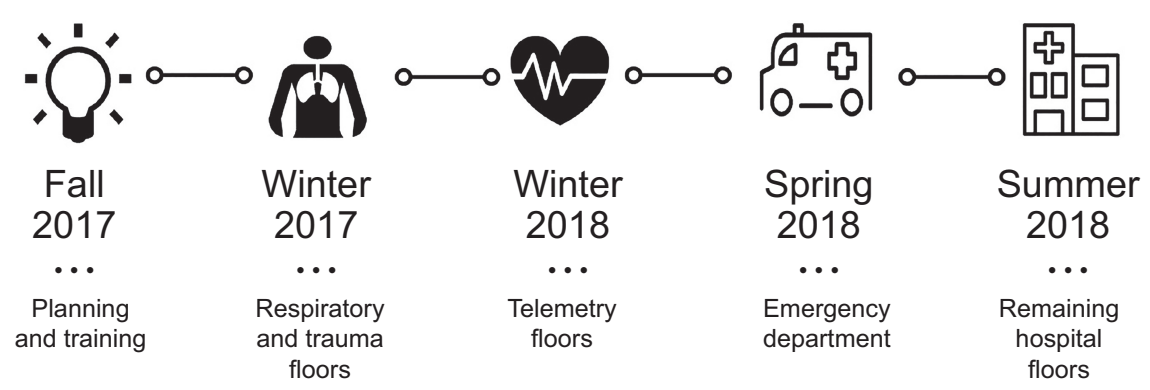

Fig. 1. Implementation timeline.

compatible with use on the patient floors. The hospital had 40 total devices available across all hospital locations during the study period, with a typical case load of 20 25 units running at one time. Another device (Optiflow, Fisher \& Paykel) was also used at the study hospital, but use of this device was limited to the ICU because general in-patient rooms did not have the capacity to support this device.

While the primary purpose of this study is to share and describe the HFNC education and implementation process at our hospital, we also present patient demographics and outcomes from the implementation period for the cohort as a whole. This cohort study is descriptive in nature, and data are used to supplement the description of the implementation process and to present a transparent summary of implementation successes and failures.

\section{Implementation Strategy}

In September 2017, a multidisciplinary study team was formed to develop a comprehensive implementation strategy for using HFNC outside the ICU. The study team included representatives from the pulmonology, trauma, emergency medicine, and respiratory therapy departments. The study team met at least monthly for the duration of the project.

The implementation strategy included 4 components. First, a protocol was written to guide the initiation and management of HFNC therapy. This specified the clinical indications and contraindications for HFNC and established the process for device set-up and management of patients while on HFNC therapy. The protocol (see the supplementary materials at http://www.rcjournal.com) could be applied to patients with undifferentiated respiratory compromise and increased oxygen requirements. Of note, the protocol did not require HFNC patients to receive telemetry monitoring or continuous pulse oximetry. In June 2019, a weaning procedure was added to the protocol (see the supplementary materials at http://www.rcjournal.com). This weaning procedure was implemented after the study period and is not included in the current data analysis.
Second, the implementation strategy included a plan to provide education to hospital staff. Key groups included respiratory therapists; internal medicine and surgery residents; internal medicine, pulmonology, trauma, cardiology, and emergency medicine physicians; and nurses on all patient floors and in the emergency department. While HFNC therapy was available to all patients regardless of admitting service, the protocol required that a critical care service (ie, pulmonology or trauma) was the admitting or consulting service for all floor patients to assess for appropriate use of HFNC and to manage patients receiving the therapy.

Third, the protocol required a respiratory therapist to evaluate the patient at least every $4 \mathrm{~h}$ while on HFNC, and those assessments were included in the electronic medical record as patient notes. Every assessment included patient vitals (breathing frequency, heart rate, $\left.\mathrm{S}_{\mathrm{pO}_{2}}\right)$, device settings $\left(\mathrm{F}_{\mathrm{IO}_{2}}\right.$, flow), respiratory pattern, breath sounds, modified Borg dyspnea scale score, ${ }^{36}$ comfort level with the device, ${ }^{37}$ and skin assessment (ears, nose, cheeks). In the initial evaluation, the therapist also noted whether the patient used supplemental oxygen at home. The focus on evaluation and documentation ensured that assessments were standardized across patients and floors and stored in a location in the electronic medical record that was visible to the entire care team. HFNC therapy was monitored by bedside nurses as part of normal standard of care; interval assessments were completed by respiratory therapists, and device adjustments were made by respiratory therapists under the guidance of pulmonology or trauma providers.

Fourth, the study team established a regular review of patient safety and adverse events. The team met monthly to review the implementation progress, adjust the protocol and education process, and discuss adverse events potentially linked to HFNC. This included review of all rapid-response team activations and mortalities. Medical codes for cardiac or respiratory arrest were reported to the respiratory therapy manager and reviewed in real time to ensure that the event was not associated with failure of HFNC therapy.

As shown in Figure 1, HFNC was implemented on the respiratory and trauma floors in Winter 2017, followed by 


\begin{tabular}{|c|c|c|c|}
\hline & Physicians and Residents & Nurses & Respiratory Therapists \\
\hline Theory and physiology of HFNC therapy & $\mathrm{X}$ & $\mathrm{X}$ & $\mathrm{X}$ \\
\hline HFNC protocol & $\mathrm{X}$ & $\mathrm{X}$ & $X$ \\
\hline HFNC device set-up and electronic medical record documentation & & & $\mathrm{X}$ \\
\hline HFNC device maintenance & & $\mathrm{X}$ & $\mathrm{X}$ \\
\hline De-escalation and weaning & $\mathrm{X}$ & $X$ & $X$ \\
\hline
\end{tabular}

telemetry floors. In Spring 2018, HFNC was implemented in the emergency department. HFNC was fully implemented on all in-patient hospital floors by Summer 2018.

\section{Staff Education}

It was recognized early that adequate staff education and buy-in was essential for adopting HFNC into patient care in non-ICU settings. Lack of familiarity with the protocol or the device could lead to resistance in adopting HFNC, and there was particular concern about complicating hospital bed flow if staff were uncomfortable caring for a patient receiving HFNC therapy on a general in-patient floor. Table 1 shows a breakdown of education component by audience. All groups received standardized education on the differences between traditional oxygen therapy and HFNC therapy, indications and contraindications for patient selection, and physiology and mechanics of the HFNC device. Respiratory therapists received additional training in management of the HFNC device and documentation in the electronic medical record.

The largest group to train was nursing because it involved every floor of the hospital, with the exception of the labor and delivery ward. Immediately prior to implementation of HFNC on each floor, a team from the respiratory therapy department attended staff meetings, provided educational opportunities at shift changes, and met 1-on-1 with nurses. Trainings were led by the respiratory therapy manager or supervisor with the cooperation of the nurse manager on each floor. As part of the education process, each nurse was also given the opportunity to try HFNC therapy to better understand what patients would experience on the therapy; disposables were donated to the study hospital by the device manufacturer to aid in this component of training.

\section{Data Collection and Variables}

During initial phases of implementation (Winter 2017), nurses were given an anonymous paper survey prior to training to evaluate familiarity and comfort with HFNC and to ascertain perceptions of how the device would impact patient care (see the supplementary materials at http:// www.rcjournal.com). The surveys were also used to explore areas of concern and to identify points of emphasis for education. Respondents were excluded from analysis if they failed to complete both sides of the survey.

All subjects were identified prospectively if they received HFNC for any reason during the study period (December 2017 to May 2019). For the current analysis, we included subjects who received HFNC for undifferentiated respiratory compromise if HFNC was initiated or discontinued in the emergency department or on an in-patient floor. We did not include patients who initiated and discontinued HFNC in the ICU (eg, the entire duration of therapy was in the ICU). If subjects received HFNC during more than 1 hospital encounter, only the first encounter is included in analyses.

Demographic variables included subject age and sex. We also tracked emergency department disposition, location in the hospital where HFNC was initiated and discontinued, hospital and ICU length of stay, and duration of HFNC therapy. The primary outcomes of interest were adverse events, including unplanned admission to the ICU from the floor for any reason, a rapid-response team activation on the floor while on the therapy, and in-hospital mortality (all causes). We also ascertained escalation of care from HFNC immediately to noninvasive ventilation or mechanical ventilation; this ex-cluded patients with suspected or diagnosed obstructive sleep apnea who received positive airway pressure at night per out-patient regimen and returned to HFNC in the morning.

\section{Statistical Analysis}

All analyses were performed with SPSS Basic Statistics for Windows 20.0 (IBM, Armonk, New York). Descriptive statistics were examined and reported for continuous data as means $\pm \mathrm{SD}$ or medians and interquartile ranges (IQR). Categorical data were reported as counts and percentages. To compare duration of HFNC therapy between subjects who escalated to noninvasive or mechanical ventilation and those who did not, differences between medians were calculated using the Kruskal-Wallis one-way analysis of variance. 
Table 2. Pre-training Survey Responses

\begin{tabular}{|c|c|c|c|}
\hline & Agree & Disagree & Unsure \\
\hline HFNC is easier to set up and use than CPAP & $39(27)$ & $15(10)$ & $91(63)$ \\
\hline HFNC is more comfortable for the patient than CPAP & $84(58)$ & $1(1)$ & $60(41)$ \\
\hline Patients requiring $>4 \mathrm{~L} / \mathrm{min}$ oxygen or with $\mathrm{F}_{\mathrm{IO}_{2}} \geq 0.50$ should be cared for in the ICU & $17(12)$ & $75(52)$ & $53(37)$ \\
\hline A patient receiving HFNC should also have continuous monitoring & $46(32)$ & $48(33)$ & $51(35)$ \\
\hline HFNC interferes with patient care, including physical therapy and mobility & $14(10)$ & $76(52)$ & $55(38)$ \\
\hline HFNC is no different than existing therapies on the floor & $40(28)$ & $51(35)$ & $54(37)$ \\
\hline HFNC would make it difficult for the patient to eat, drink, and sleep & $13(9)$ & $83(57)$ & $49(34)$ \\
\hline
\end{tabular}

Data are presented as $n(\%), n=145$ nurse respondents. Numbers may not add up to 100 due to rounding. HFNC $=$ high-flow nasal cannula

\section{Results}

\section{Staff Education}

During the study period, 450 emergency department and floor nurses received HFNC education and training, and survey data were collected from 145 nurses (32\%) in the initial months of implementation. Nurses who completed the surveys had a mean \pm SD age of $34 \pm 11 \mathrm{y}$ and $7 \pm 9 \mathrm{y}$ of experience as a nurse. Fifty-two percent of nurses $(n=76)$ held a bachelor's degree or higher. Forty-one percent $(n=59)$ of nurses were familiar with HFNC therapy, and 38\% $(n=55)$ had previously cared for a patient receiving HFNC. Nearly half of respondents $(n=70)$ indicated that they were comfortable caring for a patient on HFNC on the floor, while $33 \%$ of nurses $(n=48)$ were unsure if they felt comfortable doing so. The majority of nurses agreed that HFNC would improve patient care, but more than one third were unsure whether it would improve patient care or make patient care more difficult.

Nurses were also asked about the perceived impact of the device on patient care (Table 2). Across all questions, at least one third of nurse respondents were unsure as to how the therapy would directly impact patients. Most nurses agreed that patients on HFNC could be cared for on the floor and did not require an ICU bed, but only $33 \%$ of nurses believed that patients receiving HFNC on the floor did not require continuous monitoring.

\section{Frequency of Use in Hospital}

During the implementation period, 998 unique patients received HFNC across all locations of the hospital, and 346 unique subjects (32\%) met study inclusion criteria for initiation or discontinuation of therapy outside the ICU (Fig. 2). As shown in Table 3, median (IQR) subject age was 69 y (5980 ), but the full range of ages was 18-101 y. Slightly more than half of subjects $(n=182)$ were male. Overall, 171 subjects (49\%) started HFNC therapy in the emergency department, and a small number $(n=5)$ discharged from the

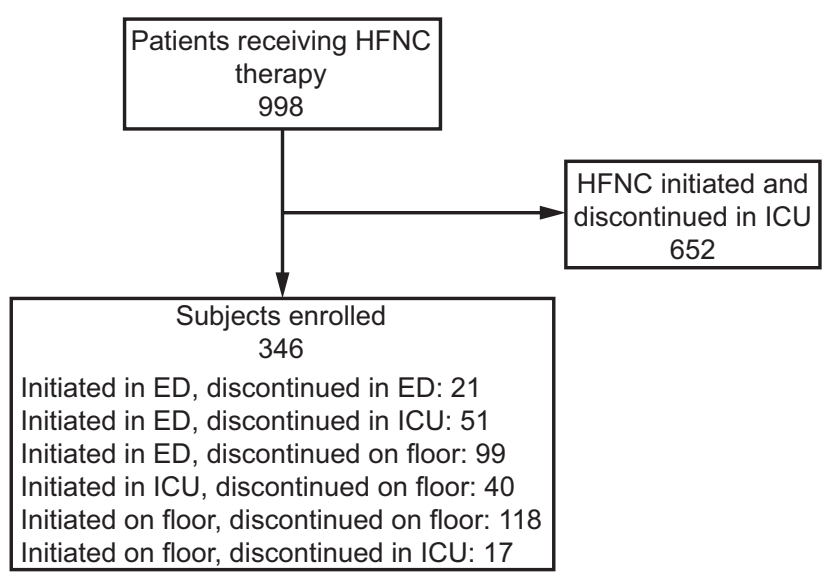

Fig. 2. Flow chart. Data do not include subjects who received HFNC during multiple encounters. $\mathrm{HFNC}=$ high-flow nasal cannula, $\mathrm{ED}=$ emergency department.

emergency department without hospital admission. Fifty-three percent of subjects $(n=184)$ avoided the ICU during hospitalization. More than two thirds of subjects $(n=238)$ received the duration of HFNC therapy in the emergency department or on the in-patient floor, which was the equivalent of 486 total hospital days of HFNC delivered outside the ICU.

Median (IQR) length of hospitalization was $8 \mathrm{~d}$ (4-12), and HFNC therapy lasted a median (IQR) of $44 \mathrm{~h}(18-90)$. Eleven percent of subjects experienced a rapid response team activation while on HFNC, and overall mortality was $13 \%$ $(n=44)$. In addition, 20 subjects $(6 \%)$ escalated therapy from HFNC to noninvasive ventilation, while 17 subjects (5\%) escalated from HFNC to mechanical ventilation. Subjects who escalated from HFNC to a higher level of support (ie, noninvasive ventilation or mechanical ventilation) had a shorter duration of HFNC therapy than those who did not transition to noninvasive or mechanical ventilation (13 vs $47 \mathrm{~h}, P<.001)$. Two subjects had a code blue called while receiving HFNC therapy on the floor and one subject had a cardiac arrest; after review of the medical record, it was determined that the codes were related to underlying medical 


\section{IMPLEMENTING HFNC OUTSIDE THE ICU}

Table 3. Subject Demographics and Outcomes

\begin{tabular}{lc}
\hline \hline Age, $y$ & $69(59-80)$ \\
Male & $182(53)$ \\
Any days in ICU & $162(47)$ \\
Emergency department disposition to home & $5(1)$ \\
Therapy delivery & \\
$\quad$ Start and stop in emergency department & $21(6)$ \\
$\quad$ Start and stop on floor & $118(34)$ \\
$\quad$ Start in emergency department, stop in ICU & $51(15)$ \\
$\quad$ Start in emergency department, stop on Floor & $99(29)$ \\
$\quad$ Start in ICU, stop on floor & $40(12)$ \\
$\quad$ Start on floor, stop in ICU & $17(5)$ \\
Adverse events & $63(18)$ \\
$\quad$ Unplanned ICU admission for any reason & $20(6)$ \\
$\quad$ Escalation of care from HFNC to noninvasive ventilation & $17(5)$ \\
Escalation of care from HFNC to invasive ventilation & $38(11)$ \\
Rapid response team activation while on HFNC & $44(13)$ \\
$\quad$ Mortality, all causes & $8(4-12)$ \\
Hospital length of stay, d & $0(0-3)$ \\
ICU length of stay, d & $44(18-90)$ \\
Duration of HFNC, h & \\
\hline Data are presented as median (interquartile range) or $n$ (\%). $n=346$ subjects. Numbers may not \\
add up to 100 due to rounding. \\
HFNC = high-flow nasal cannula \\
\hline
\end{tabular}

comorbidities, not HFNC therapy. Both subjects were transferred to the ICU and required mechanical ventilation, but family elected for comfort care and the subjects died.

\section{Discussion}

Initiating a new respiratory therapy is a challenge for any hospital, particularly when it involves a device that has historically only been available in the ICU. To maximize staff comfort and acceptance of HFNC therapy, it is essential to consider and plan for staff education as part of the implementation process. Study findings indicate that a robust and systematic implementation plan led to widespread adoption of HFNC outside the ICU setting. Most importantly, implementing HFNC on in-patient floors was deemed safe and efficacious. Even though $11 \%$ of subjects had a change in physiologic status while receiving HFNC therapy that prompted a rapid-response team activation, review of these incidents indicated increased vigilance on the part of bedside nurses who followed the protocol and activated the team at the first sign of subject deterioration. In addition, incidence of escalation of care to mechanical ventilation was $5 \%$ and mortality was $13 \%$, which were lower than the previous respective rates of $13 \%$ and $21 \%$ at our institution $^{17}$ and lower than rates in comparable literature. ${ }^{32}$ Subjects who escalated therapy also had a significantly shorter duration of HFNC therapy than those who did not, indicating that the team was aggressive and did not delay definitive care. The device was utilized for all ages of adult patients for any condition that resulted in hypoxic respiratory failure.

More than half of the sample avoided the ICU during hospitalization, which amounted to 486 hospital days of HFNC delivered outside the ICU. Prior to this protocol, HFNC was only available in the ICU, so patients who required the therapy for hypoxia but were otherwise hemodynamically stable required an ICU bed to receive therapy. National estimates show that an ICU day is almost 3 times as expensive as a day on the in-patient floor of the hospital, ${ }^{9,38,39}$ so this effort not only maximized bed flow but it also lowered health care costs for the subject and the hospital. In addition, 40 subjects (12\%) began HFNC in the ICU and transitioned to the floor while receiving therapy. This indicates that HFNC therapy was not an impediment to transfer and that patients could leave the ICU when they were otherwise stable, even if they were still receiving HFNC therapy.

Subjects with no ICU days had a lower mortality rate than subjects who spent $\geq 1 \mathrm{~d}$ in the ICU (7\% vs $13 \%$ ) and never escalated from HFNC to noninvasive ventilation. In the context of a robust HFNC protocol with appropriate patient selection, many patients can receive the therapy outside the ICU and can do so without increased risk to their safety or well-being. This optimizes hospital resources, including ICU beds, for patients with more critical conditions.

Preimplementation survey results also indicated that while most nurses were amenable and comfortable with adopting HFNC into practice on their floors, one third of nurses were uncertain about the efficacy of the device and their role in managing patients receiving HFNC therapy. Face-to-face interaction with respiratory therapy staff, as well as the opportunity to try the device themselves, may have mitigated this discomfort. Widespread acceptance was evidenced by increasing requests for the therapy from staff on floors that did not yet have the therapy. As this occurred, the implementation timeline was accelerated.

\section{HFNC Barriers and Challenges}

As with any effort that transforms patient care, there were barriers and challenges that were overcome during the course of implementation. First, most nurses were trained and educated prior to the device being available on their floor, but turnover in nursing staff required constant and continuous education and monitoring. New nursing staff were trained at the bedside when their first patient was started on HFNC. In addition, interval documentation by respiratory therapists was monitored in real time to ensure that all critical values were documented appropriately; the respiratory therapy manager intervened and re-educated staff who were inconsistent or incomplete in their electronic medical record electronic medical record documentation.

Second, as HFNC became more accepted in the hospital, new hospital locations were requested (eg, in-patients 


\section{IMPLEMENTING HFNC OUTSIDE THE ICU}

receiving HFNC in the out-patient dialysis ward, pre- and postbronchoscopy, and in postoperative recovery). In these circumstances, additional training was extended to nursing staff to ensure that patients could initiate or continue to receive HFNC therapy in these locations. When new locations or indications were requested, the study team reviewed the protocol and made revisions as appropriate.

Third, there are several times during hospitalization when patients must be transported, the first of which is when a patient is moved from the ambulance to the emergency department. Because emergency medical services in the metropolitan area of the study hospital do not currently utilize HFNC in ambulances, many patients with hypoxic respiratory failure arrived at the hospital on a CPAP device or a non-rebreather face mask. To assist these subjects, HFNC was initiated after arrival at the study hospital while the subject was still in the ambulance via a portable HFNC device, and then the subject was moved to an emergency department bed. It is also important that HFNC subjects continued to ambulate and receive therapies during hospitalization; therefore, a portable setup was used in the hospital to mobilize and transport subjects between areas of care. Each subject's oxygen requirements determined the volume and mode for transport, but subjects were transported with the HFNC device when possible. One exception was when a subject went to the magnetic resonance imaging suite, at which time they were temporarily transitioned to a nonrebreather face mask. If the subject could not tolerate the non-rebreather face mask, they received noninvasive ventilation during imaging. Of note, the HFNC device was adapted to include a battery backup that was sufficient for most transport within the hospital.

Fourth, once the HFNC protocol was implemented hospital-wide and was embraced by all floors and specialties, it became clear that clinicians and staff needed a more robust process for weaning from the HFNC device. There is no published literature or guideline for this process. The study team examined data from the current sample to estimate the settings at discontinuation of therapy, and a conservative weaning procedure was added to the HFNC protocol. The respiratory therapy team began weaning by first decreasing oxygen $\left(\mathrm{F}_{\mathrm{IO}_{2}}\right)$ and then flow, both of which were done incrementally based on subject condition. Future analysis is warranted to determine if the weaning procedure affected duration of therapy or patient outcomes.

Fifth, the HFNC protocol at the study hospital does not exclude patients who are receiving tube feedings, and there were no events during the study period where a subject failed therapy due to aspiration. There is no published evidence that HFNC impedes feeding or swallowing, with some evidence that HFNC may even enhance the swallowing function ${ }^{40}$ but this is an area that would benefit from more clinical research.
Finally, in the early phase of protocol implementation, 4 subjects developed skin breakdown behind the ears due to HFNC tubing being secured too tightly on the subject's head. Two of these subjects developed a pressure ulcer. This issue was addressed immediately by revising the protocol to require documentation of skin checks at every respiratory therapy assessment (ie, every 4 h). Nurses were re-educated on the issue, and any concerns about skin integrity were reported immediately to the respiratory therapy leadership and bedside nurse, who would evaluate the subject collaboratively to address the problem. If a subject had known skin integrity problems, the care team enlisted the help of the wound team to monitor and prevent skin breakdown. Of note, the HFNC cannulas are latex-free, therefore the skin issues were not caused by an allergic reaction to the cannula.

\section{Study Limitations}

This study has several limitations. First, all subjects were identified prospectively and analyzed retrospectively, so there are inherent limitations in documentation and the variables available through chart abstraction. In addition, there was no standardized measure of patient acuity across diagnoses. Second, the study sample is heterogeneous in diagnosis, and the protocol is standard of care at the study hospital; therefore subjects are not randomized to treatment groups and there is no concurrent comparison group available for analysis. Results cannot be generalized to specific diagnoses or patient populations, but future analyses will examine subpopulations in comparison to historical controls. Third, the postimplementation nurse survey had a very low response rate $(n=$ 40 ), and data were not sufficiently generalizable to the nursing population as a whole. Preimplementation surveys were administered in-person prior to HFNC training, which generated a high response rate; however, postimplementation surveys were administered via e-mail and electronic survey software, and we could not generate a sufficient response rate, despite repeated requests for participation.

\section{Conclusions}

While HFNC therapy has traditionally been limited to the ICU setting, HFNC can be safely initiated in locations outside the ICU if delivered within the context of a comprehensive and collaborative implementation plan that emphasizes staff education, quality review, and a robust protocol. Our results indicate that, with appropriate patient selection, many patients can safely and effectively receive HFNC without spending any days in the ICU. 


\section{IMPLEMENTING HFNC OUTSIDE THE ICU}

\section{ACKNOWLEDGMENTS}

The authors thank Dr Daniel Dodge, Elizabeth Fuchsen, Keith Lamb, and Dr Sheryl Sahr for assistance in study design. The authors also thank the Vice President of Medical Affairs, the Chief Nursing Executive, nursing administrative leadership, and nurse managers on the floor for their support and commitment to this process.

\section{REFERENCES}

1. Blakeman T. Evidence for oxygen-use in the hospitalized patient: is more really the enemy of good? Respir Care 2013;58(10):1679-1693.

2. Kim LHY, Chu DK, Alhazzani W. Rethinking oxygen therapy for hospitalized patients. Ann Intern Med 2019;171(6):HO2-HO3.

3. MacIntyre NR. Supporting oxygenation in acute respiratory failure. Respir Care 2013;58(1):142-150.

4. Kallet RH, Diaz JV. The physiologic effects of noninvasive ventilation. Respir Care 2009;54(1):102-115.

5. Vestbo J, Hurd SS, Agustí AG, Jones PW, Vogelmeier C, Anzueto A, et al. Global strategy for the diagnosis, management, and prevention of chronic obstructive pulmonary disease: GOLD executive summary. Am J Respir Crit Care Med 2013;187(4):347-365.

6. Pancioli AM, Bullard MJ, Grulee ME, Jauch EC, Perkis DF. Supplemental oxygen use in ischemic stroke patients: does utilization correspond to need for oxygen therapy? Arch Intern Med 2002;162(1):49-52.

7. Stockinger ZT, Mcswain NE, Jr. Prehospital supplemental oxygen in trauma patients: its efficacy and implications for military medical care. Mil Med 2004;169(8):609-612.

8. Papazian L, Corley A, Hess D, Fraser JF, Frat J-P, Guitton C, et al. Use of high-flow nasal cannula oxygenation in ICU adults: a narrative review. Intensive Care Med 2016;42(9):1336-1349.

9. Halpern NA, Pastores SM. Critical care medicine beds, use, occupancy, and costs in the United States: a methodological review. Crit Care Med 2015;43(11):2452-2459.

10. Wallace DJ, Seymour CW, Kahn JM. Hospital-level changes in adult ICU bed supply in the United States. Crit Care Med 2017;45(1):e67-e76.

11. Kahn JM, Rubenfeld GD, Rohrbach J, Fuchs BD. Cost savings attributable to reductions in intensive care unit length of stay for mechanically ventilated patients. Med Care 2008;46(12):1226-1233. [Database]

12. Kaier K, Heister T, Motschall E, Hehn P, Bluhmki T, Wolkewitz M. Impact of mechanical ventilation on the daily costs of ICU care: a systematic review and meta regression. Epidemiol Infect 2019;147:e314.

13. Reardon PM, Fernando SM, Van Katwyk S, Thavorn K, Kobewka D, Tanuseputro $\mathrm{P}$, et al. Characteristics, outcomes, and cost patterns of high-cost patients in the intensive care unit. Crit Care Res Pract 2018;2018:5452683.

14. Frat JP, Thille AW, Mercat A, Girault C, Ragot S, Perbet S, et al. High-flow oxygen through nasal cannula in acute hypoxemic respiratory failure. N Engl J Med 2015;372(23):2185-2196.

15. Gaunt KA, Spilman SK, Halub ME, Jackson JA, Lamb KD, Sahr SM. High-flow nasal cannula in a mixed adult ICU. Respir Care 2015;60 (10):1383-1389.

16. Hernández G, Vaquero C, Colinas L, Cuena R, Gonzalez P, Canabal A, et al. Effect of postextubation high-flow nasal cannula vs noninvasive ventilation on reintubation and postextubation respiratory failure in high-risk patients: a randomized clinical trial. JAMA 2016;316 (15):1565-1574.

17. Lamb KD, Spilman SK, Oetting TW, Jackson JA, Trump MW, Sahr SM. Proactive use of high-flow nasal cannula with critically ill subjects. Respir Care 2018;63(3):259-266.

18. Monro-Somerville T, Sim M, Ruddy J, Vilas M, Gillies MA. The effect of high-flow nasal cannula oxygen therapy on mortality and intubation rate in acute respiratory failure: a systematic review and meta-analysis. Crit Care Med 2017;45(4):e449-e456.

19. Messika J, Ahmed KB, Gaudry S, Miguel-Montanes R, Rafat C, Sztrymf B, et al. Use of high-flow nasal cannula oxygen therapy in subjects with ARDS: a 1-year observational study. Respir Care 2015;60(2):162-169.

20. Nagata K, Morimoto T, Fujimoto D, Otoshi T, Nakagawa A, Otsuka $\mathrm{K}$, et al. Efficacy of high-flow nasal cannula therapy in acute hypoxemic respiratory failure: decreased use of mechanical ventilation. Respir Care 2015;60(10):1390-1396.

21. Nedel WL, Deutschendorf C, Moraes Rodrigues Filho E. High-flow nasal cannula in critically ill subjects with or at risk for respiratory failure: a systematic review and meta-analysis. Respir Care 2017;62 (1): $123-132$.

22. Nishimura M. High-flow nasal cannula oxygen therapy in adults: physiological benefits, indication, clinical benefits, and adverse effects. Respir Care 2016;61(4):529-541.

23. Roca O, Hernández G, Díaz-Lobato S, Carratalá JM, Gutiérrez RM, Masclans JR, Spanish Multidisciplinary Group of High Flow Supportive Therapy in Adults (HiSpaFlow). Current evidence for the effectiveness of heated and humidified high flow nasal cannula supportive therapy in adult patients with respiratory failure. Crit Care 2016;20(1):109.

24. Yuste ME, Moreno O, Narbona S, Acosta F, Peñas L, Colmenero M. Efficacy and safety of high-flow nasal cannula oxygen therapy in moderate acute hypercapnic respiratory failure. Rev Bras Ter Intensiva 2019;31(2):156-163.

25. Chanques G, Constantin JM, Sauter M, Jung B, Sebbane M, Verzilli D, et al. Discomfort associated with underhumidified high-flow oxygen therapy in critically ill patients. Intensive Care Med 2009;35(6):996-1003.

26. Mauri T, Turrini C, Eronia N, Grasselli G, Volta CA, Bellani G, et al. Physiologic effects of high-flow nasal cannula in acute hypoxemic respiratory failure. Am J Respir Crit Care Med 2017;195(9):1207-1215.

27. Betters KA, Gillespie SE, Miller J, Kotzbauer D, Hebbar KB. High flow nasal cannula use outside of the ICU; factors associated with failure. Pediatr Pulmonol 2017;52(6):806-812.

28. Dadlez NM, Esteban-Cruciani N, Khan A, Shi Y, McKenna KJ, Azzarone G, et al. Safety of high-flow nasal cannula outside the ICU for previously healthy children with bronchiolitis. Respir Care 2019;64(11):1410-1415.

29. Jones PG, Kamona S, Doran O, Sawtell F, Wilsher M. Randomized controlled trial of humidified high-flow nasal oxygen for acute respiratory distress in the emergency department: the HOT-ER study. Respir Care 2016;61(3):291-299.

30. Lenglet H, Sztrymf B, Leroy C, Brun P, Dreyfuss D, Ricard JD. Humidified high flow nasal oxygen during respiratory failure in the emergency department: feasibility and efficacy. Respir Care 2012;57 (11):1873-1878.

31. Rittayamai N, Tscheikuna J, Praphruetkit N, Kijpinyochai S. Use of high-flow nasal cannula for acute dyspnea and hypoxemia in the emergency department. Respir Care 2015;60(10):1377-1382.

32. Zemach S, Helviz Y, Shitrit M, Friedman R, Levin PD. The use of high-flow nasal cannula oxygen outside the ICU. Respir Care 2019;64 (11):1333-1342.

33. Darling E, Searles B. Oxygenator change-out times: the value of a written protocol and simulation exercises. Perfusion 2010;25(3):141-143.

34. Greiner AC, Knebel E. Health professions education: a bridge to quality. Washington DC: National Academies Press; 2003.

35. Halub ME, Spilman SK, Gaunt KA, Lamb KD, Jackson JA, Oetting TW, et al. High-flow nasal cannula therapy for patients with blunt thoracic injury: a retrospective study. Can J Respir Ther 2016;52(4):110-113.

36. Kendrick KR, Baxi SC, Smith RM. Usefulness of the modified 0-10 Borg scale in assessing the degree of dyspnea in patients with COPD and asthma. J Emerg Nurs 2000;26(3):216-222. 


\section{IMPLEMENTING HFNC OUTSIDE THE ICU}

37. Maggiore SM, Idone FA, Vaschetto R, Festa R, Cataldo A, Antonicelli F, et al. Nasal high-flow versus Venturi mask oxygen therapy after extubation. effects on oxygenation, comfort, and clinical outcome. Am J Respir Crit Care Med 2014;190(3):282-288.

38. Gooch RA, Kahn JM. ICU bed supply, utilization, and health care spending: an example of demand elasticity. JAMA 2014;311(6):567-568.
39. Halpern NA, Pastores SM. Critical care medicine in the United States 2000-2005: an analysis of bed numbers, occupancy rates, payer mix, and costs. Crit Care Med 2010;38(1):65-71.

40. Sanuki T, Mishima G, Kiriishi K, Watanabe T, Okayasu I, Kawai M, et al. Effect of nasal high-flow oxygen therapy on the swallowing reflex: an in vivo volunteer study. Clin Oral Invest 2017;21(3):915-920.

This article is approved for Continuing Respiratory Care Education credit. For information and to obtain your CRCE

(free to AARC members) visit

www.rcjournal.com 